\title{
Osteossarcomas humanos de alto grau: imunoexpressão de p53, erb-2 e P-glicoproteína, e correlação com o parâmetro anaplasia
}

\author{
P-glycoprotein, erb2 and p53 expression in high-grade human osteosarcomas \\ and their correlation with anaplasia
}

\begin{abstract}
Maria Teresa de Seixas Alves'; Luciana Nakao Odashiro Miiji'; Larissa Cardoso Marinho'; Antonio Sergio Petrilli;; Reynaldo Jesus-Garcia ${ }^{3}$; Silvia Caminada Tolledo4; Francy Reis da Silva Patricio ${ }^{1}$
\end{abstract}

\begin{abstract}
unitermos
Osteossarcoma

Imuno-histoquímica

Tumores ósseos

Neoplasias ósseas

Marcadores histológicos

\section{resumo}

Introdução: Osteossarcoma (OS), o mais freqüente tumor primário maligno do osso, tem comportamento local agressivo e alto índice de disseminação sistêmica. Os eventos que permitem o crescimento e a disseminação tumoral ainda permanecem controversos. Os estudos sobre a carcinogênese e a progressão dessa neoplasia, com base na imunoexpressão de c-erb-B2, P-glicoproteína (P-gp) e p53, apresentam resultados conflitantes acerca do real valor prognóstico e suas correlações com parâmetros histologicos. A anaplasia, em neoplasias na infância, constitui parâmetro histológico de agressividade tumoral e quimiorresistência. Nos OS primários ou metastáticos, seu significado permanece controverso. Por outro lado, em outras neoplasias humanas, a expressão do c-erb-B2 relaciona-se com p53, grau nuclear e outros parâmetros de agressividade. Objetivo: Avaliar a imunoexpressão de p53, c-erb-B2 e P-gp em OS, correlacionando os parâmetros entre si e com a presença de anaplasia. Método: O estudo incluiu 96 biópsias pré-quimioterapia de pacientes com OS de alto grau, diagnosticados entre 1991 e 2000. A pesquisa imuno-histoquímica de p53, P-gp e c-erb-B2 foi feita pela técnica da estreptoavidina-biotina-peroxidase. Foram considerados positivos os casos onde havia imunoexpressão em $10 \%$ ou mais das células neoplásicas. Somente colorações membranosa (para cerb-B2 e P-gp) e nuclear (para p53) foram consideradas positivas. Anaplasia foi definida como no tumor de Wilms, sendo considerada presente ou ausente. Resultados: Anaplasia pôde ser avaliada em 82/96 casos, estando presente em 29 (35,36\%). Imunoexpressão de p53 foi detectada em 25 dos 60 casos (36,23\%); de P-gp, em 30 dos 73 casos (41,1\%); e de c-erb-B2, em 22 dos 55 casos (40\%). Os resultados demonstraram associação entre as imunoexpressões de c-erb-B2 e p53 $(p=0,042)$, p53 e o parâmetro anaplasia $(p=0,015)$, anaplasia e Pg $(p=0.034)$ Conclusões: A imunoexpressão de $\mathrm{p53}$, c-erb-B2 e P-gp é evento relativamente freqüente em OS de alto grau, metastáticos e não-metastáticos ao diagnóstico. Os resultados reforçam a hipótese de que nessa neoplasia na presença de anaplasia ocorrem simultaneamente eventos adversos, que atuam conjuntamente. A anaplasia constitui marcador histológico do status da P-gp e/ou do p53 em parte dos OS de alto grau e nestes talvez seja indicativa de quimiorresistência. Não houve associação positiva entre p53 e P-gp.
\end{abstract}

Background: Osteosarcomas (OS), the most frequent primary malignant bone tumors, have aggressive local behavior and high rate of metastatization. The events that allow tumor growth and dissemination are still controversial. The studies about carcinogenesis and tumor progression in this neoplasia, which are based on c-erb-B2, P-glycoprotein $(P-g p)$ and $p 53$ immunoexpression, show conflicting results as to the real prognostic value and its correlations with histological parameters. Anaplasia in childhood neoplasias is a histological parameter of tumor aggressiveness and chemoresistance. In primary or metastatic OS, its meaning remains controversial. On the other hand, in other human neoplasias, c-erb-B2 expression is associated with $p 53$, nuclear grade and other aggressiveness parameters. Objective: The aim of the present study was to evaluate $553, c$-erb-B2 and P-gp immunoexpression in OS, correlating the parameters with the presence of anaplasia. Methods: This study included 96 pre-chemotherapy biopsies in patients with high-grade OS diagnosed between 1991 and 2000. The immunohistochemical evaluation of 553 and $c$-erb-B2 was carried out with the streptavidin-biotin-peroxidase technique. Cases were considered positive when there was immunoexpression in 10\% or more neoplastic cells. Only membrane staining (for c-erb-B2 and P-gp), and nuclear staining (for p53) were considered positive. Anaplasia was defined as Wilms' tumor, and considered present or absent. Results: Anaplasia was present in 29 out of 82 cases (35.36\%); p53 immunoexpression was detected in 25 out of 60 cases (36.23\%); P-gp, in 30 out of 73 cases (41.1\%); and c-erb-B2, in 22 out of 55 cases (40\%). The results demonstrated an association between $c-e r b-B 2$ and $p 53$ immunoexpression $(p=0.042)$, $p 53$ and parameter of anaplasia $(p=0.015)$, anaplasia and $P$-gp $(p=0.034)$. Conclusions: The $p 53, c-e r b-B 2$ and $P$-gp immunoexpression is relatively frequent in high-grade, metastatic and non-metastatic OS. The results reinforce the hypotheses that in the presence of anaplasia adverse events may occur simultaneously in this neoplasm. Anaplasia may become a histological marker for P-gp and/or 553 status in some high-grade OS, and it may indicate chemoresistance. There was no positive association between $p 53$ and P-gp. key words

Osteosarcoma

Immunohistochemistry

Bone tumor

Bone neoplasm

Histological markers

1. Departamento de Patologia da Universidade Federal de São Paulo (UNIFESP).

2. Departamento de Pediatria da UNIFESP.

3. Departamento de Ortopedia da UNIFESP.

4. Departamento de Genética do Instituto de Oncologia Pediátrica/Crupo de Apoio ao Adolescente e Criança com Câncer da UNIFESP (IOP/GRAACC/ UNIFESP) 


\section{Introdução}

Tumores ósseos primários representam $8,5 \%$ de todos os cânceres na infância e adolescência, sendo a maior parte deles osteossarcomas (OS). Apesar da quimioterapia agressiva e do tratamento cirúrgico, esses tumores exibem significativo índice de disseminação sistêmica ${ }^{(1)}$

O estadiamento ao diagnóstico é o principal determinante da sobrevida. Outros parâmetros de valor prognóstico incluem o local do tumor, tamanho, resposta à quimioterapia e remissão cirúrgica ${ }^{(6,7,10,17,34,41,50)}$.

O desenvolvimento de resistência à quimioterapia pelas células tumorais é evento crítico bem conhecido pelo oncologista, que pode surgir em qualquer momento do tratamento. Esse evento (multi drug resistance [MDR]) pode ser estudado por muitas metodologias, incluindo a imunoexpressão de uma proteína de alto peso molecular de 170-180 quilodálton $(\mathrm{kDa})$, presente nas membranas celulares, denominada P-glicoproteína (P-gp). Essa glicoproteína é capaz de deslocar agentes quimioterápicos, como a doxorrubicina, para fora da célula por meio de canais de $\mathrm{Ca}^{++}$, podendo causar ainda resistência cruzada a drogas não relacionadas, afetando o prognóstico e a incidência de recaída tumoral após o tratamento inicial.

Há anticorpos monoclonais contra essa glicoproteína que podem ser utilizados em material fixado em formalina e incluído em parafina ${ }^{(43,52,54)}$. Em OS existem resultados divergentes quando diferentes metodologias e anticorpos são utilizados para estudar esse evento e sua correlação com o prognóstico $(4,8,22,37,36,44,46,47,49)$. Inativação do gene supressor de tumor p53 é o mais freqüente evento genético em cânceres humanos. Esse gene é denominado "guardião do genoma", pois mantém a estabilidade genômica em muitas situações, incluindo hipoxia, dano ao DNA e ativação de oncogenes ${ }^{(14,55)}$.

Mutações que ocasionam defeitos no gene p53 podem representar etapas iniciais da carcinogênese em tumores ósseos e de partes moles e/ou determinar o comportamento biológico do desenvolvimento tumoral. A imunoexpressão aumentada da proteína p53 geralmente é resultado de mutações gênicas, com produção de proteína anômala, estável, com meia-vida prolongada. A superexpressão nuclear do p53 é evento comum no OS, mas seu significado clínico é ainda desconhecido e provavelmente sem implicações prognósticas, quando estudado isoladamente. Entretanto, na avaliação do prognóstico o tipo específico de mutação, não somente a mutação em si, e outros mecanismos envolvidos na carcinogênese do OS devem ser analisados em conjunto(27, 38, 48, 53).
O proto-oncogene c-erbB2 (também denominado neu ou HER-2) localiza-se no cromossomo humano 17 na banda q21. Ele codifica receptor transmembrana de $185 \mathrm{kDa}$, que exibe significativa similaridade estrutural ao receptor do fator de crescimento epidérmico (Epidermal growth factor receptor [EGFR] $)^{(1)}$. Apesar da semelhança estrutural com o receptor de EGF, a proteína ErbB2 não se liga a esse receptor.

Acredita-se que o gene codifique um importante fator de crescimento que controla o crescimento do epitélio fetal(28). Esse receptor parece ter atividade de tirosinoquinase como um domínio intracelular, o qual, uma vez ativado pelo seu ligante extracelular, leva à indução de cascata de mecanismos responsáveis pelo crescimento celular. Em câncer de mama, a superexpressão do HER-2 ocorre em 15\%-40\% e está associada com piores índices de sobrevida e a evento metastático ${ }^{(29)}$.

Onda et al. ${ }^{(31)}$ foram os primeiros a relatar correlação entre o OS e a expressão de HER-2. Os autores encontraram que a aparente expressão de ErbB2 está correlacionada a uma diminuição de sobrevida. Após esse trabalho inicial, outros autores relataram a expressão de c-erbB2 em OS, com diferentes metodologias e resultados divergentes, inclusive quanto a real presença de coloração membranosa e seu papel no evento metástase. Apesar de os dados avaliáveis serem limitados, especialmente quando comparados ao câncer de mama, estudos-piloto estão sendo conduzidos, utilizando trastuzumabe em pacientes com OS recidivados, que superexpressaram HER-2 ${ }^{(18,20)}$.

A anaplasia em tumor de Wilms encontra-se significativamente associada a pior prognóstico e má resposta à quimioterapia ${ }^{(5)}$. O mesmo foi observado em rabdomiossarcomas ${ }^{(16,21)}$. Osteossarcoma de subtipo histológico anaplásico é raro, mas subtipos convencionais podem apresentar células anaplásicas, tanto no tumor primário como nas metástases. A proposta deste estudo é avaliar a imunoexpressão de p53, c-erb-B2 e P-gp em OS humanos, com ênfase na presença de anaplasia.

\section{Materiais e métodos}

Estudamos biópsias de 96 pacientes com OS de alto grau, diagnosticados entre 1991 e 2000, no Departamento de Patologia da Universidade Federal de São Paulo/Escola Paulista de Medicina (UNIFESP/EPM). Em relação ao subtipo histológico, utilizou-se a classificação da Organização Mundial da Saúde (OMS) ${ }^{(42)}$. Anaplasia nuclear foi definida de maneira similar ao tumor de Wilms ${ }^{(5)}$ e classificada como presente ou ausente (Figuras $\mathbf{1}$ e $\mathbf{2}$ ). 


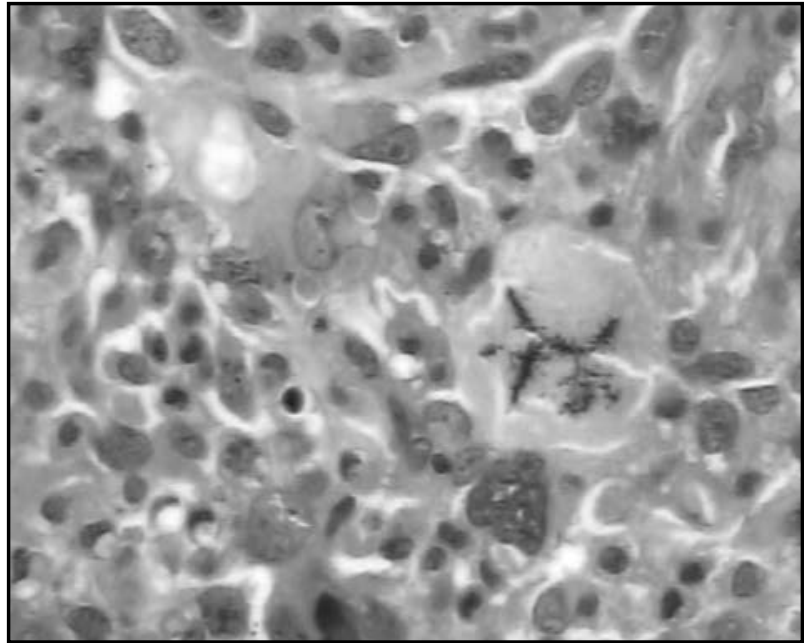

Figura 1 - Mitose multipolar: parâmetro para anaplasia em osteossarcoma (hematoxilina-eosina [HE] 400x)

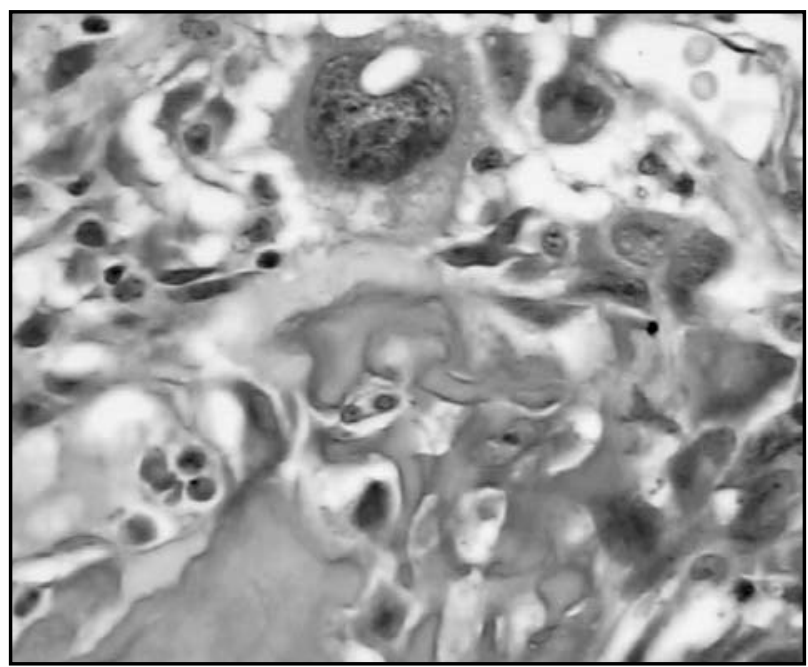

Figura 2 - Cariomegalia acentuada: achado anaplásico (HE 400x)

A imunoexpressão da P-gp, cerb-B2, p53 e suas correlações com parâmetros histológicos foi avaliada. Os casos foram classificados como positivos e negativos, quando a expressão era observada em $10 \%$ ou mais das células tumorais. Somente coloração de membrana (completa ou incompleta) foi considerada positiva para cerb-B2 (Figura 3). Apenas coloração nuclear para p53 foi considerada positiva (Figura 4), bem como coloração membranosa para P-gp (Figura 5).

Havia 55 pacientes do sexo masculino $(57,29 \%)$ e 41 do feminino (42,71\%); a idade variou de 5 a 74 anos (média de 17,73 anos; mediana de 15,38 anos). O local primário foi o fêmur em 46 (47,92\%); tíbia em 35 (36,46\%); úmero em 5 (5,21\%); fíbula em 3 (3,13\%); em outros sítios, em 7 casos ( $1,04 \%$ cada). Havia 89 (92,71\%) OS nos membros e $7(7,29 \%)$ no tronco.

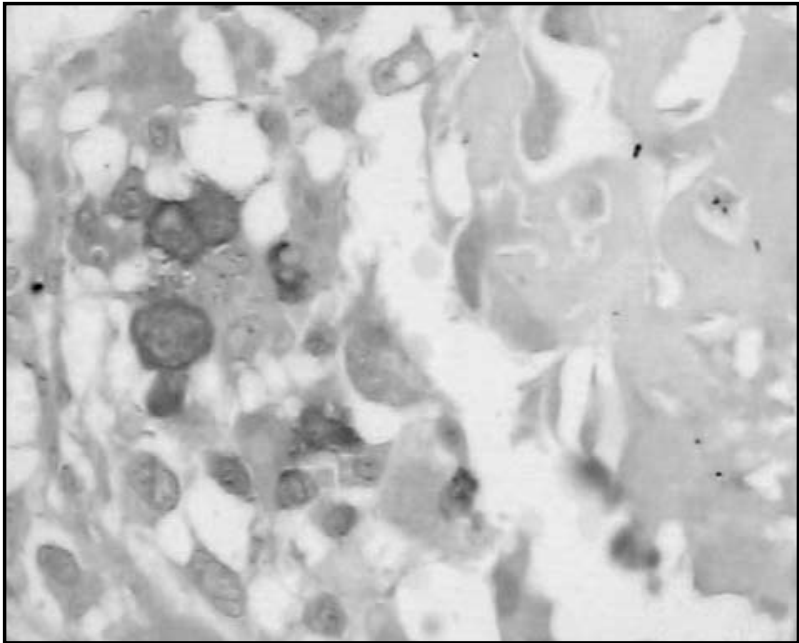

Figura 3 - c- erb-B2: expressão membranosa e citoplasmática em osteossarcomas (IH 400x)

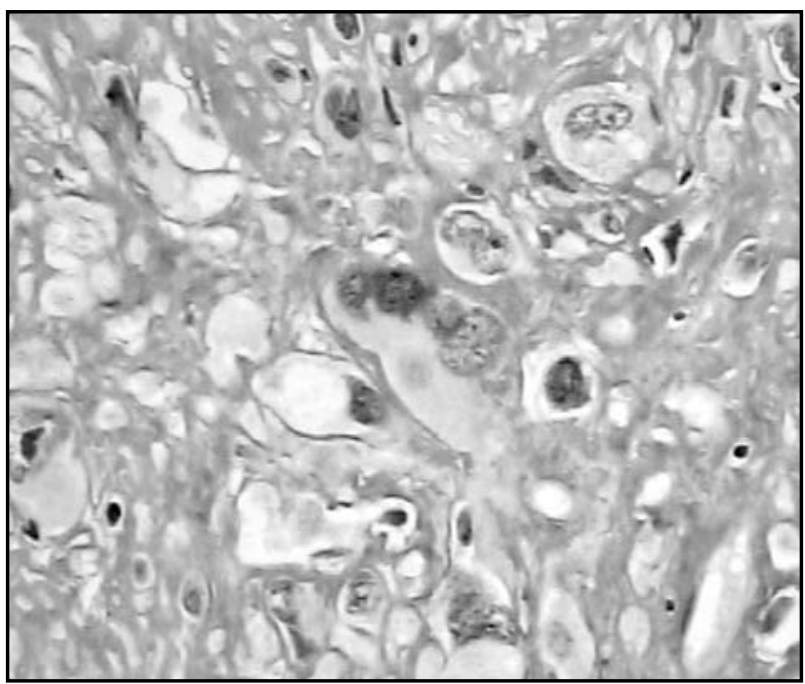

Figura 4 - p53: expressão nuclear em osteossarcoma (IH 400x)

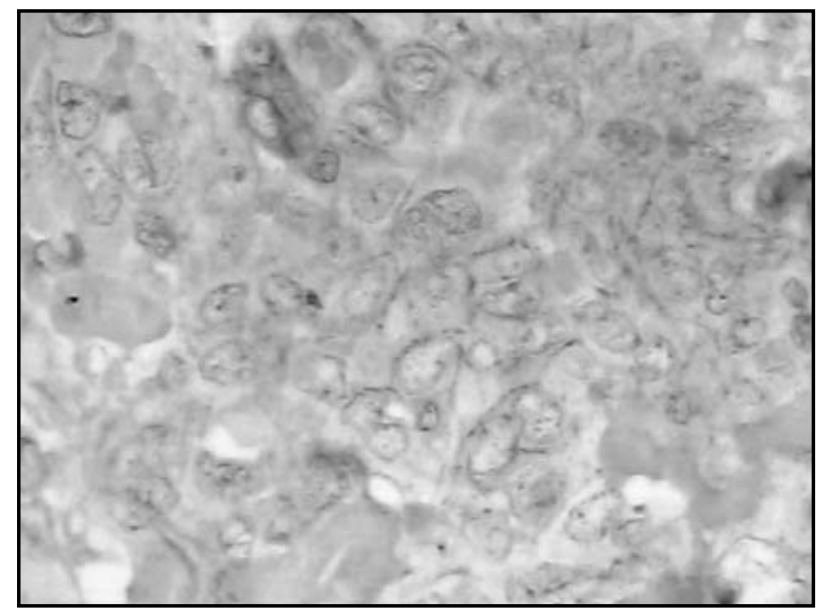

Figura 5 - P-glicoproteína : expressão membranosa em osteossarcoma (IH 400x) 


\section{Método imuno-histoquímico}

Todas as biópsias dos 96 pacientes avaliados foram submetidas à análise imuno-histoquímica $(\mathrm{IH})$, a partir dos blocos de parafina. $\mathrm{O}$ material foi descalcificado utilizando-se ácido nítrico $\left(\mathrm{NHO}_{3}\right)$ a 7,5\%. $\mathrm{O}$ método $\mathrm{IH}$ foi realizado em cortes histológicos de $4 \mu \mathrm{m}$ de espessura, em lâminas previamente silanizadas. A recuperação antigênica (HIER) foi realizada em forno de microondas (MW) na potência de 700 watts, com imersão das lâminas em solução-tampão de citrato de sódio (citrato de sódio $10 \mathrm{mM} \mathrm{pH}$ 6), em duas sessões de 9 minutos cada, após o que se resfriaram as lâminas à temperatura ambiente por pelo menos $20 \mathrm{~min}$.

Realizou-se o bloqueio da peroxidase endógena utilizando-se peróxido de hidrogênio a 3\%, imergindo as lâminas cinco vezes por 5 min cada. Foram utilizados os anticorpos monoclonais anti-humano p53 (clone DO7, Dako A/S Denmark, 1:20) e anti-humano P-gp (clone C494, Dako Corporation USA, 1:600) e o anticorpo policlonal anti-cerb-B2 oncoproteína humana (AO 485, DakoA/S Denmark, 1:500). Os anticorpos primários foram aplicados diluídos em soroalbumina bovina (BSA) a $1 \%$ nas concentrações referidas anteriormente, sendo incubados pernoite em câmara úmida a $4^{\circ} \mathrm{C}$. Em seguida, foram lavadas em solução salina tamponada de fosfatos (PBS, pH 7,4) por $30 \mathrm{~min}$, sendo colocado o reagente número 1 do kit LSAB/HRP (Dako A/S K0690). Após 30 min, a $37^{\circ} \mathrm{C}$, as lâminas foram lavadas novamente em PBS e incubadas por mais 30 min no reagente 2 do kit LSAB/HRP.

A reação final foi revelada imergindo as lâminas em 3,3-diaminobenzidina tetra-hidroclorido, na presença de $0,05 \%$ de peróxido de hidrogênio $\left(\mathrm{H}_{2} \mathrm{O}_{2}\right)$ em PBS por 5 min a $37^{\circ} \mathrm{C}$. A seguir, as lâminas foram lavadas em água corrente e contracoradas pela hematoxilina de Harris por 5 min, lavadas em água amoniacal, desidratadas em concentrações crescentes de álcool, imersas em xilol e montadas utilizando meio de montagem rápido (Entellan). Rim normal, adenocarcinoma colônico e de mama foram utilizados como controles positivos para P-gp, p53 e erbB2 respectivamente. Controles negativos foram obtidos retirando-se o anticorpo primário da reação.

\section{Análise estatística}

O teste exato de Fisher foi utilizado para analisar as associações entre os parâmetros imuno-histoquímicos e anaplasia.

\section{Resultados}

Anaplasia foi detectada em 29 de 82 casos (35,36\%). Em 14 casos, o parâmetro não pode ser avaliado pela presença de artefatos técnicos de esmagamento ou devido à representatividade tumoral da amostra. $O$ estudo imunohistoquímico revelou imunoexpressão de p53 em 25 de 69 casos (36,23\%); P-gp em 30 dos 73 casos (41,10\%); e c-erb-B2, em 22 dos 55 casos (40\%). Não houve associação entre a expressão de $\mathrm{p} 53$ e P-gp $(p=0,461)$; P-gp e c-erb-B2 $(p=0,782)$; e c-erb-B2 e anaplasia $(p=0,382)$. Houve associação entre p53 e anaplasia $(p=0,015) ;$ P-gp e anaplasia $(p=0,034)$; e $p 53$ e c-erb-B2 $(p=0,042)$

(Tabelas 1 a 4).

\section{Discussão}

A metástase ao diagnóstico é o principal parâmetro para o prognóstico em OS de alto grau. O tamanho tumoral constitui-se em outra variável que tem sido significativamente associada ao prognóstico por muitos autores $(6,7,34,50)$.

Tabela 1 Associações imuno-histoquímicas do parâmetro p-glicoproteína

\begin{tabular}{lccc}
\hline Variáveis & $\begin{array}{c}\text { Negativa } \\
n(\%)\end{array}$ & $\begin{array}{c}\text { p-glicoproteína } \\
\text { Positiva } \\
n(\%)\end{array}$ & $\begin{array}{c}\text { Teste exato de Fisher } \\
\text { (valor de } p)\end{array}$ \\
$\begin{array}{l}\text { p53 } \boldsymbol{n}=\mathbf{6 9} \\
\text { Positivo }\end{array}$ & $12(48)$ & $17(52)$ & 0,461 \\
$\begin{array}{l}\text { Negativo } \\
\text { c-erbB2 } \boldsymbol{i = 5 5}\end{array}$ & $27(61,36)$ & $17(38,64)$ & \\
Positivo & & $10(45,45)$ & 0,782 \\
Negativo & $12(54,55)$ & $13(39,39)$ & \\
\hline
\end{tabular}


Tabela 2 Associações imuno-histoquímicas do parâmetro p53

\begin{tabular}{|c|c|c|c|}
\hline \multirow[t]{2}{*}{ Variáveis } & \multicolumn{2}{|c|}{ p53 } & \multirow{2}{*}{$\begin{array}{l}\text { Teste exato de Fisher } \\
\text { (valor de } p \text { ) }\end{array}$} \\
\hline & $\begin{array}{c}\text { Negativo } \\
n(\%)\end{array}$ & $\begin{array}{c}\text { Positivo } \\
n(\%)\end{array}$ & \\
\hline \multicolumn{4}{|c|}{ C-erb-B2 $(n=54)$} \\
\hline Positivo & $11(30,56)$ & $11(61.11)$ & 0,042 \\
\hline Negativo & $25(69,44)$ & $7(38,89)$ & \\
\hline \multicolumn{4}{|c|}{ P-glicoproteína $(n=69)$} \\
\hline Presente & $17(38,64)$ & $12(48)$ & 0,461 \\
\hline Ausente & $27(61,36)$ & $13(52)$ & \\
\hline
\end{tabular}

Tabela 3 Associações entre os parâmetros imuno-histoquímicos e anaplasia

\begin{tabular}{lccc}
\hline Variáveis & $\begin{array}{c}\text { Anaplasia } \\
\text { Presente }\end{array}$ & $\begin{array}{c}\text { Ausente } \\
n(\%)\end{array}$ & $\begin{array}{c}\text { Teste exato de Fisher } \\
\text { (valor de } p)\end{array}$ \\
$\begin{array}{l}\text { p-glicoproteína }(\boldsymbol{n}=\mathbf{6 5}) \\
\text { Positiva }\end{array}$ & $14(51,85)$ & $13(48,15)$ & 0,034 \\
$\begin{array}{l}\text { Negativa } \\
\text { p53 }(\boldsymbol{n}=64)\end{array}$ & $9(23,68)$ & $2976,32)$ & \\
Positivo & $13(56,52)$ & $10(43,48)$ & 0,015 \\
Negativo & $10(24,39)$ & $31(75,61)$ & \\
C-erb-B2 $(\boldsymbol{n}=\mathbf{5 5 )}$ & & & 0,382 \\
Positivo & $09(40,91)$ & $13(59,09)$ & \\
Negativo & $09(27,27)$ & $24(72,73)$ & \\
\hline
\end{tabular}

Tabela 4 Associações imuno-histoquímicas do parâmetro c-erb-B2

\begin{tabular}{lccc}
\hline Variáveis & $\begin{array}{c}\text { Presente } \\
n(\%)\end{array}$ & $\begin{array}{c}\text { c-erb-B2 } \\
\text { Ausente } \\
n(\%)\end{array}$ & $\begin{array}{c}\text { Teste exato de Fisher } \\
\text { (valor de } p)\end{array}$ \\
$\begin{array}{l}\text { p-glicoproténa }(\boldsymbol{n}=\mathbf{5 5}) \\
\text { Positiva }\end{array}$ & $10(45,45)$ & $13(39,39)$ & 0,782 \\
$\begin{array}{l}\text { Negativa } \\
\text { p53 }(\boldsymbol{n}=\mathbf{5 4 )}\end{array}$ & $12(54,55)$ & $20(60,61)$ & \\
Positivo & $11(61,11)$ & $7(38,89)$ & 0,042 \\
Negativo & $11(30,56)$ & $25(69,44)$ & \\
\hline
\end{tabular}

Bieling et $a l^{(7)}$ recomendam ser melhor a mensuração do volume tumoral. Para Bielack et al. ${ }^{(6)}$, um tumor é considerado grande quando seu tamanho for pelo menos um terço do comprimento do osso envolvido. Acreditamos ser necessário homogeneizar a análise do tamanho, também levando em conta a idade dos pacientes.

Atualmente, graças às novas abordagens ortopédicas, tem sido possível aumentar o número de cirurgias conser- vadoras, propiciando melhor qualidade de vida e integração social. A superexpressão de uma série de oncogenes e cariótipos complexos, caracterizados primariamente por múltiplas anormalidades estruturais não-balancedas tem sido relatada em OS ${ }^{(53)}$. Entretanto, se essas alterações genéticas são capazes de predizer o prognóstico dos pacientes ainda não está claro, bem como as vias de iniciação, progressão e potencial metastático. Entre esses oncogenes, o gene 
supressor p53 tem se mostrado alterado quando estudado por meio de diferentes metodologias $(5,11,13,19,22,24,27,30,53$, 56). Optamos por utilizar o clone DO7 anti p53 por ser o mais sensível(22), porém muitos autores encontraram valores variando de $12 \%$ a $67 \%$ de imunoexpressão $0^{(3,5,38)}$.

Neste estudo encontramos $36,23 \%$ de coloração nuclear, valor intermediário dos encontrados na literatura. Provavelmente, a alta sensibilidade do clone DO7, a utilização de HIER e o fato de que se utilizaram somente espécimes pré-tratamento foram responsáveis pelo alto índice. Há muitos trabalhos com diferentes métodos de avaliação, o que pode explicar os resultados divergentes. Não houve associação entre o status do p53 e a expressão de P-gp.

De modo similar ao nosso estudo, Serra et al. ${ }^{(45)}$ não encontraram associação entre p53 e P-gp. Tem sido sugerido que as alterações no p53 constituem-se em importantes eventos na tumorigênese. Em nossos casos detectamos alto nível de expressão de $p 53$, presente em $36,23 \%$, com grande número de células imunocoradas e P-gp, suportando a hipótese de que as alterações do p53 são eventos comuns nos OS de alto grau.

Overholtzer et al. ${ }^{(32)}$ concluíram que as mutações no p53 podem ser responsáveis pela instabilidade genômica freqüentemente observada nos OS. Por outro lado, acreditamos que a progressão tumoral nos OS de alto grau constitui-se em evento de múltiplas etapas, envolvendo alterações gênicas, como do p53, proliferação, habilidade para metastatizar, associada com resistência à quimioterapia, como evento primário ou adquirido.

O método IH é o mais comumente utilizado para detectar superexpressão do HER-2/neu em material de arquivo anatomopatológico. Entretanto, os resultados IH podem ser influenciados por alterações antigênicas induzidas pelos métodos de fixação e descalcificação, bem como pela habilidade em resgatar os antígenos. A sensibilidade e especificidade dos diferentes anticorpos variam de $6 \%$ $80 \%{ }^{(9)}$. Contudo, a interpretação dos resultados é variável em muitas das casuísticas, sendo somente o padrão de coloração membranosa considerado específico para o HER-2 e correlacionado com a expressão gênica ${ }^{(15)}$.

Contrariamente, como ocorre em muitos tumores, como carcinomas nasofaríngeo, colorretal e papilífero de tireóide, tem sido relatada positividade citoplasmática com significado prognóstico ${ }^{(2,40,44)}$. Entretanto, falsa positividadade pode ocorrer por reatividade cruzada com o epitopo C219 da glicoproteína, que é um homólogo para p185c erbB2 ${ }^{(23)}$.
Neste estudo, foi demonstrada imunoexpressão de c-erb-B2 em 40\% dos casos analisados, como igualmente foi relatado por Gorlick et al.(12). Observamos também positividade citoplasmática e em células gigantes de tipo osteoclasto. Achados similares foram descritos por outros autores, como Onda et al. ${ }^{(31)}$.

Maitra et al. ${ }^{(25)}$, Kilpatrick et al. ${ }^{(20)}$ e Thomas et al. ${ }^{(51)}$ não encontraram expressão membranosa em seus casos. É importante enfatizar que diferentes metodologias e sistemas de graduação foram usados para definir a positividade para o c-erb-B2 em OS. Tal fato pode em parte explicar os resultados discrepantes.

O material analisado revelou alto nível de expressão para a P-gp $(41,10 \%)$, como também foi encontrado por outros autores ${ }^{(4,36,37)}$. A relação entre P-gp e o prognóstico em OS ainda permanece controversa ${ }^{(8,33,37,44,45,46,47,49)}$. Não observamos associação entre a imunoexpressão de P-gp, p53 e c-erb-B2. Por outro lado, detectamos associação entre a positividade de $\mathrm{P}$-gp e a presença de anaplasia ( $p=$ $0,034)$, e entre anaplasia e p53 $(p=0,015)$.

Anaplasia em tumor de Wilms e rabdomiossarcomas estão relacionados com resistência à quimioterapia e a pior prognóstico $(5,16,21)$. A associação que encontramos é particularmente interessante porque o parâmetro anaplasia pode se constituir em marcador histológico para o status da P-gp e/ou do p53 em OS.

Este estudo utilizou apenas material pré-quimioterapia e, por questões de amostragem, não foi possível pesquisar a totalidade dos marcadores em todos os blocos. Nossa análise, em casos somente de OS de alto grau, é pioneira no que se refere à casuística brasileira, enfatizando a imunoexpressão dos marcadores erb-B2, p53 e P-gp, e suas correlações com o parâmetro histológico anaplasia.

Cumpre lembrar que a associação de p53 e erb-B2 sugere efeito sinérgico na carcinogênese do OS. É extremamente importante a correlação desses achados, com parâmetros clínicos de agressividade já consagrados na literatura mundial. Nesse sentido, torna-se fundamental abordagem multiinstitucional, aumentando a casuística anatomopatológica, com uniformização de parâmetros e de conceitos, e reforçando a idéia da criação de centros de referência no Brasil.

Em conclusão, os resultados deste estudo demonstraram que os osteossarcomas de alto grau freqüentemente demonstram múltiplos eventos adversos simultâneos. 


\section{Referências}

1. AKIYAMA, T. et al. The product of the c-erbB-2 gene: a 185 kilodalton glycoprotein with tyrosine kinase activity. Science, n. 232, p. 1644-6, 1986

2. AKSLEN, L. A.; VARHAUG, J. E. Oncoprotein and tumor progression in papillary thyroid carcinoma: presence of epidermal growth factor receptor, c-erbB-2 protein, p21 ras protein, and proliferation indictors in relation to tumor recurrences and patient survival. Cancer, n. 76, p. 1643-54, 1995.

3. ANDREASSEN, A. et al. p53 abnormalities in different subtypes of human sarcomas. Cancer Research, n. 53, p. 468-71, 1993.

4. BALDINI, N. et al. Expression of P-glycoprotein in high-grade osteosarcomas in relation to clinical outcome. N Engl J Med, n. 333, p. 1380-5, 1995.

5. BECKWITH, J. B.; PALMER, N.F. Histopathology and prognosis of Wilms tumors: results from the first national Wilm's tumor study. Cancer, n. 41, p. $1937-$ 48, 1978

6. BIELACK, S. S. et al. Prognostic factors in high-grade osteosarcoma of the extremities or trunk: an analysis of 1,702 patients treated on neoadjuvant Cooperative Osteosarcoma Study Group Protocols. J Clin Oncol, n. 20, p. 776-90, 2002

7. BIELING, P. et al. Tumor size and prognosis in aggressively treated osteosarcoma. J Clin. Oncol, v. 14, p. 848-58, 1996.

8. CHAN, H. S. et al. P-glycoprotein expression: critical determinant in the response to osteosarcoma. J Natl Cancer Inst, n. 89, p. 1706-15, 1997.

9. GANCBERG, D. et al. Sensitivity of HER-2/neu antibodies in archival tissue samples of invasive breast carcinomas: correlation with oncogene amplification in 160 cases. Am J Clin Pathol, n. 113, v. 5, p. 675-82, 2000.

10. GLASSER, D.B. et al. Survival, prognosis, and therapeutic response in osteogenic sarcoma: the Memorial Hospital Experience. Cancer, n. 69, p. 698-708, 1992.

11. GOKGOZ, N. et al. Comparison of p53 mutations in patients with localized osteosarcoma and metastatic osteosarcoma. Cancer, n. 92, v. 8, p. 2181-9, 2001.

12. GORLICK, R. et al. Expression of HER2/erbB-2 correlates with survival in osteosarcoma. J Clin Oncol, n. 17, p. 2781-8, 1999

13. GRUNDMAN, E. et al. New aspects of cell biology in osteossarcoma. Path Res Pract, n. 191, p. 563-70, 1995.

14. HAINAUT, P. et al. Database of p53 gene somatic mutations in human tumors and cell lines: updated compilation and future prospects. Nucleic Acids Res, n. 25, p. 151-7, 1997.

15. HANNA, W.; KHAN, H. J.; TRUDEAU, M. Evaluation of HER-2/neu (erbB-2) status in breast cancer: from bench to bedside. Mod Pathol, n. 12, p. 827-834, 1999.

16. HAWKINS, H. K.; CAMACHO-VELASQUES, J. V. Rhabdomyosarcoma in children. Correlation of form and prognosis in one institution's experience. Am J Surg Pathol, n. 11, p. 531-42, 1987.

17. HUVOS, A.G. et al. Telangiectatic osteogenic sarcoma: a clinicopathologic study of 124 patients. Cancer, n. 49, p. 1679-89, 1982.

18. KAY, W.E. et al. Cytoplasmic c-erbB-2 protein expression correlates with survival in Duke's B colorectal carcinoma. Histopathology, n. 25, p. 455-61, 1994.

19. KAWAGUCHI, K. et al. Molecular analysis of p53, MDM2, and $\mathrm{H}$-ras genes in osteosarcoma and malignant fibrous histiocytoma of bone in patients older than 40 years. Mod Pathol, n. 15, v. 9, p. 878-88, 2002

20. KILPATRICK, S. E. et al. Clinicopathologic analysis of Her-2/neu immunoexpression among various histologic subtypes and grades of osteosarcoma. Mod Pathol, n. 14 , v. 12 , p. $1277-83,2001$

21. KODET, R. et al. Childhood rhabdomyosarcoma with anaplastic (pleomorphic) features: a report of the Intergroup Rhabdomyosarcoma study. Am J Surg Pathol, n. 17, p. 443-53, 1996.

22. LEE, P. D. et al. Quantitative analysis of multidrug resistance gene expression in human osteosarcomas. Br J Cancer, n. 74, p. 1046-50, 1996.

23. LIU, B. et al. Cross-reactivity of C219 anti-p170 (MDR-1) antibody with p185 (c-erbB2) in breast cancer cells: cautions on evaluating p170 (MDR-1). J Natl Cancer Inst, n. 89, v. 20, p. 1524-9, 1997.

24. LONARD0, F. et al.. p53 and MDM2 alterations in osteossarcomas. Cancer, n. 79, p. 1541-7, 1997.

25. MAITRA, A. et al. Amplification of Her-2/neu oncogene is uncommon in pediatric osteosarcoma. Cancer, n. 92, p. 677-83, 2001.

26. MEYERS, P.A. ; GORLICK, R. Osteosarcoma. Pediatric Clin North Am, v. 44, p. 973-89, 1997.

27. MILLER, C. W. et al. Alterations of the p53, RB and MDM2 genes in osteossarcoma. J Cancer Res Clin Oncol, n. 122, p. 559-65, 1996.

28. MORI, S. et al. C-2rbB-2 gene product, a membrane protein commonly expressed on human fetal epithelial cells. Lab Invest, n. 61, p. 93-7, 1989.

29. MORRIS, C. D. et al. Human epidermal growth factor receptor 2 as a prognostic indicator in osteogenic sarcoma. Clin Orthop, n. 382, p. 59-65, 2001.

30. OLIVEIRA, P. et al. Analysis of p53 expression in osteosarcoma of the jaws: correlation with clinicopathologic and DNA ploidy findings. Human Pathol, n. 28, p. 1361-5, 1997.

31. ONDA, M. et al. ErbB-2 expression is correlated with poor prognosis for patients with osteosarcoma. Cancer, n. 77, p. 71-8, 1996.

32. OVERHOLTZER, M. et al. The presence of p53 mutations in human osteosarcomas correlates with high levels of genomic instability. Proc Natl Acad Sci USA, n. 100, v. 20, p. 11547-52, 2003.

33. PARK, Y. B. et al. The co-expression of p53 protein and $\mathrm{P}$-glycoprotein is correlated to a poor prognosis in osteosarcoma. Int Orthop, n. 24, v. 6, p. 307-10, 2001.

34. PETRILLI, A. S. et al. IIB Osteosarcoma Current management, local control, and survival statistics, São Paulo, Brazil. Clinical Orthopaedics, n. 270, p. 60-6, 1991. 
35. PETRILLI, A. S. et al. Brazilian Osteosarcoma Treatment Group Studies III and IV. Results of the Brazilian Osteosarcoma Treatment Group Studies III and IV: prognostic factors and impact on survival. J Clin Oncol, n. 24, v. 7, p. 1161-8, 2006.

36. PÖLS, M. et al. P-glycoprotein expression in high grade central osteosarcoma and normal bone cells: an immunohistochemical study. Gen Diagn Pathol, n. 142, p. 317-25, 1996.

37. RADIG, K. et al. Expression of p-glycoprotein in high grade osteosarcomas with special emphasis on chondroblastic subtype Gen. Diagn Pathol, n. 143, p. 1-13, 1997.

38. RADIG, K. et al. Mutation spectrum of p53 gene in highly malignant human osteossarcomas. Gen Diagn Pathol, n. 142, p. 25-32, 1996.

39. ROSEN, G. et al. Preoperative chemotherapy for osteogenic sarcoma: selection of postoperative adjuvant chemotherapy based on the response of primary tumor to preoperative chemotherapy. Cancer, n. 49, p. 122130, 1982.

40. ROYCHOWDHURY, D. F. et al. New prognostic factors in nasophrayngeal carcinoma: tumor angiogenesis and cerbB-2 expression. Cancer, n. 77, p. 1419-26, 1996.

41. SAETER, G. et al. Systemic relapse of patients with osteogenic sarcoma prognostic factors for long term survival. Cancer, n. 75, p. 1084-93, 1995.

42. SCHAJOWICZ, F.; SISSONS, H. A.; SOBIN, L. H. The World Health Organization's Histologic Classification of Bone Tumors: a commentary on the second edition. Cancer, n. 75. p. 1208-14, 1995.

43. SCHEPER, R. J. et al. Monoclonal antibody JSB-1 detects a highly conserved epitope on the p-glycoprotein associated with multi-drug-resistance. Int J Cancer, n. 42, p. 389-94, 1988.

44. SCHWARTZ, C. L. et al. Multiple drug resistance in osteogenic sarcoma: INT0133 from the Children's Oncology Group. J Clin Oncol, n. 25, v. 15, p. $2057-$ 62, 2007.

45. SERRA, M. et al. Relationship between P-glycoprotein expression and p53 status in high-grade osteosarcoma. Int J Oncol, n. 14, v. 2, p. 301-7, 1999.

46. SERRA, M. et al. May P-glycoprotein status be used to stratify high-grade osteosarcoma patients? Results from the Italian/Scandinavian Sarcoma Group 1 treatment protocol. Int J Oncol, n. 29, v. 6, p. 1459-68, 2006.

47. SHNYDER, S. D. et al. P-glycoprotein and metallothionein expression and resistance to chemotherapy in osteosarcoma. Br J Cancer, n. 78, p. 757-9, 1998.

48. SLOMINSKI, A. et al. Molecular pathology of soft tissue and bone tumors: a review. Arch Pathol Lab Med, n. 123, p. 1246-59, 1999.

49. STEIN, U. etal. Expression of the MDR1 gene in bone and soft tissue sarcomas of adult patients. Eur J Cancer, n. 29, p. 1979-81, 1993.

50. TAYLOR, W. F. et al. Prognostic variables in osteosarcoma: a multi-institutional study. J Natl Cancer Inst, n. 81, p. 21-30, 1989.

51. THOMAS, D. G. et al. Absence of HER2/neu gene expression in osteosarcoma and skeletal Ewing's sarcoma. Clin Canc Res, n. 8, p. 788-93, 2002.

52. TOTH, K. et al. New immunohistochemical "Sandwich" staining method for MDR1 p-glycoprotein detection with JSB-1 monoclonal antibody in formalin-fixed, paraffin-embedded human tissues. Am J Pathol, n. 144, p. 227-36, 1994.

53. UEDA, Y. et al. Analysis of mutant p53 protein in osteosarcomas and other malignant and benign lesions of bone. J Cancer Res Clin Oncol, n. 119, p. 172-8, 1993.

54. VERGIER, B. et al. Expression of MDR1/P-glycoprotein in human sarcomas. Br. J. Cancer, n. 68, p. 1221-6, 1993.

55. VOGELSTEIN, B.; LANE, D.; LEVINE, A. J. Surfing the p53 network. Nature, n. 408, p. 307-10, 2000.

56. YOKOYAMA, R. et al. Clinicopathologic implications of MDM2, p53 and K-ras gene alterations in osteosarcomas: MDM2 amplification and p53 mutations found in progressive tumors. Pathol Res Pract, n. 194, v. 9, p. 615-21, 1998. 